\title{
Prostate Cancer Pathologic Stage pT2b (2002 TNM Staging System): Does It Exist?
}

\author{
Maisa M. Quintal, Luis A. Magna, Marbele S. Guimaraes, Thais Ruano, Ubirajara Ferreira, \\ Athanase Billis
}

Department of Anatomic Pathology (MMQ, MSG, TR, AB), Department of Medical Genetics and Biostatistics (LAM), and Department of Urology (UF), School of Medicine, State University of Campinas (Unicamp), Campinas, SP, Brazil

\begin{abstract}
Objective: In the 1997 TNM staging system, tumors were classified into a single subdivision: T2a, and bilateral tumor involvement (T2b). In the 2002 TNM staging system, tumors are subclassified as T2a (less than one half of one lobe involvement), T2b (more than one half of one lobe involvement), and T2c (bilateral involvement). A recent study questioned the existence of a true pathologic pT2b tumor. The aim of our study is to verify this question.

Materials and Methods: The study population consisted of 224 men submitted to radical retropubic prostatectomy. The surgical specimens were histologically evaluated by complete embedding and whole-mount processing. Tumor extent was evaluated by a point-count method. The surgical specimens were staged according to the 2002 TNM staging system.

Results: Using the 2002 TNM criteria, the surgical specimens were classified as pT2a, 28 (12.50\%); pT2b, 0 (0\%); pT2c, 138 (61.61\%); pT3a, 30 (13.39\%); and, pT3b, 28 (12.50\%). Using the point-count method for tumor extent evaluation, the minimum and maximum total points obtained in unilateral tumors were 192 and 368 points, respectively; the most extensive unilateral tumor showed 68 positive points (less than half the minimum total point-count).

Conclusions: Using the point-count method for tumor extent, our study questions a real existence for pathologic stage pT2b tumors (unilateral tumors involving greater than one-half of one lobe).
\end{abstract}

Key words: prostate neoplasms; carcinoma; pathology; prostate-specific antigen Int Braz. J Urol. 2006; 32: 43-7

\section{INTRODUCTION}

In the 1997 TNM staging system, unilateral disease was combined into a single subdivision, T2a, and bilateral tumor involvement as $\mathrm{T} 2 \mathrm{~b}$ (1). In the 2002 TNM staging system (2) tumors were subclassified as T2a (one half of one lobe involvement or less), T2b (more than half of one lobe involvement, but not both lobes), and T2c (involvement of both lobes). A recent study questioned the existence of a true pathologic pT2b tumor (3). The purpose of our study is to check this question.

\section{MATERIALS AND METHODS}

The study was done on 224 consecutive patients submitted to radical retropubic prostatectomy from January 1997 to June 2005 in our Institution. The clinicopathologic variables studied were age, 
preoperative PSA, prostate weight, Gleason score, tumor extent, extraprostatic extension, seminal vesicle invasion, and surgical margins.

The surgical specimen previously fixed was weighed, measured and the entire surface inked. The bladder neck and apical margins were amputated. From each cone-shaped amputated margins, 8 fragments were processed through perpendicular sections relative to the margins. The rest of the prostate was serially cut in transverse sections at 3 to $5 \mathrm{~mm}$ intervals. The prostate slices were subdivided into quadrants and labeled to allow reconstruction as wholemount sections.

Blocks were embedded in paraffin, cut at $6 \mu \mathrm{m}$, and one section from each block was stained with hematoxylin and eosin. Presence of adenocarcinoma was diagnosed according to the criteria of Mostofi and Price (4). Histological grading was performed according to the Gleason system $(5,6)$. Seminal vesicle invasion was defined as invasion of the muscular wall as described by Epstein et al. (7), and extraprostatic extension was diagnosed according to Bostwick \& Montironi (8) whenever cancer was seen in adipose tissue. Positive surgical margins (bladder, apical or circumferential) were defined as cancer cells touching the inked surface of the prostate.

Tumor extent was estimated by use of a pointcount method $(9,10)$. Drawn on a sheet of paper, each quadrant of the whole-mount sections contained 8 equidistant points. During the microscopic examination of the slides, the tumor area was drawn on the correspondent quadrant seen on the paper. At the end of the examination, the amount of positive points represented an estimate of tumor extent (Figure-1).

\section{RESULTS}

Table-1 shows the whole-mount surgical specimens characteristics of 224 patients serially submitted to retropubic prostatectomy. The mean age was 63.35 years (range, 43-76 years); mean preoperative PSA $10.23 \mathrm{ng} / \mathrm{mL}$ (range, 0.28-50); and, mean prostate weight $39.36 \mathrm{~g}$ (range, 15-130g). Using the 2002 TNM pathologic classification, 28 specimens were pT2a $(12.50 \%) ; 138$, pT2c $(61.61 \%) ; 30$, pT3a

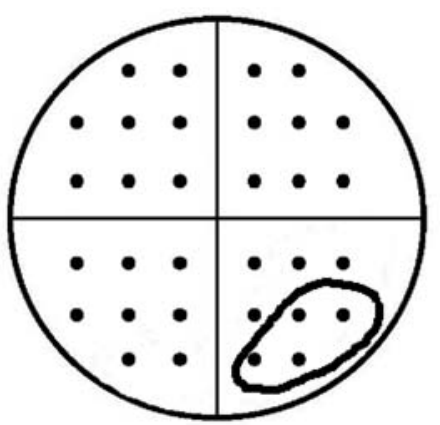

Figure 1 - Point-count method for tumor extent evaluation. Transverse whole section of a prostate with a total of 32 points. Tumor extent corresponds to 4 positive points in the lower-right quadrant.

Table 1 - Patient and tumor characteristics.

\begin{tabular}{ll}
\hline Characteristics & \multicolumn{1}{c}{$\mathbf{N}(\boldsymbol{\%})$} \\
\hline Mean age in years (range) & $63.35(43-76)$ \\
Mean preoperative PSA ng/mL (range) & $10.23(0.28-50)$ \\
Mean prostate weight in grams (range) & $39.36(15-130)$
\end{tabular}

2002 TNM pathologic classification

pT2a

pT2b

$28(12.50)$

pT2c

pT3a

$138(61.61)$

pT3b

$30(13.39)$

$28(12.50)$

Gleason score

4-6

87 (38.84)

7

$124(55.36)$

8-10

$13(5.80)$

Extraprostatic extension

Positive

$55(24.55)$

Negative

$169(75.45)$

Seminal vesicle invasion

Positive

$28(12.50)$

Negative

$196(87.50)$

\section{Surgical margins}

Positive

$92(41.07)$

Negative 
(13.39\%); and, 28, pT3b (12.50\%) pathologic stages. No specimen pathologic pT2b stage was found. In 87 (38.84\%), $124(55.36 \%)$, and $13(5.80 \%)$ specimens, the Gleason score was 4-6, 7 and 8-10, respectively. Extraprostatic extension was found in 55 (24.55\%); seminal vesicle invasion in $28(12.50 \%)$, and positive surgical margins in $92(41.07 \%)$ surgical specimens.

The results in pathologic stage pT2 unilateral tumors using the point-count method for extent evaluation are shown in Table-2. The mean and median of the total points were 289.33 and 300 points, respectively (range 192-368 points). The mean and median of the positive points were 9.75 and 5.5 points, respectively (range 1-68 points). The most extensive unilateral tumor showed 68 points, therefore less than half the minimum total point-count.

\section{COMMENTS}

In the fifth edition of the TNM classification of malignant tumors in 1997 (1), stage T2 of prostate carcinoma was subdivided into T2a (tumor involving one lobe) and $\mathrm{T} 2 \mathrm{~b}$ (tumor involving both lobes). In the sixth edition of 2002 (2), unilateral tumors were subclassified into T2a (one half of one lobe involvement or less), and T2b (more than half of one lobe involvement, but not both lobes).

Eichelberger \& Cheng (3) question the existence of a true pathologic stage pT2b tumor. They studied 369 prostate cancer patients treated by radical prostatectomy. Prostate cancers were multifocal in 312 cases $(85 \%)$. The majority of the specimens were pathologic stage pT2 (276, or 75\%). Using the 2002 TNM staging criteria, $54(15 \%)$ of the tumors were stage pT2a, $222(60 \%)$ were pT2c, $75(20 \%)$ were pT3a, and 18 (5\%) were pT3b. No pathologic stage $\mathrm{pT} 2 \mathrm{~b}$ tumors were identified. Our findings agree with Eichelberger \& Cheng (3). No tumor pathologic stage pT2b was found and the frequency of the stages in our series is very similar to theirs: stage pT2a, 28 (12.50\%); pT2c, 138 (61.61\%); pT3a, $30(13.39 \%)$; and, pT3b 28 (12.50\%). A higher number of cases in stage pT3b in our series probably is due to selection of patients with high level of serum
Table 2 - Total and positive points obtained in pathologic stage pT2 unilateral tumors using the point-count method for extent evaluation.

\begin{tabular}{lll}
\hline & Total Points & Positive Points \\
\hline Mean \pm SD & $289.33 \pm 52.25$ & $9.75 \pm 13.74$ \\
Median & 300.00 & 5.5 \\
Minimum & 192.00 & 1.00 \\
Maximum & 368.00 & 68.00 \\
\hline
\end{tabular}

PSA submitted to prostatectomy in 1997 and 1998. The mean preoperative PSA was 8.4 and $10.23 \mathrm{ng} /$ $\mathrm{mL}$, in Eichelberger \& Cheng's (3) and in our series, respectively.

Based on clinical characteristics there is also questioning regarding subclassification of stage $\mathrm{T} 2$. Freedland et al. (11) studied 1606 men with organconfined disease (pT2NO) who were treated with radical prostatectomy between 1982 and 2003 by one surgeon. Using the 1997 TNM staging criteria, clinical characteristics were compared between men with pT2a and pT2b tumors using rank-sum analysis, and prostate-specific antigen (PSA) recurrence data were compared using log-rank analysis. There was no difference in PSA recurrence rates between men with pT2aNO versus pT2bNO tumors. Rubin et al. (12) reported the results of 1613 consecutive radical prostatectomy cases conducted from 1994 to 2002 with up to 8 years of clinical follow-up. In this report, the authors concluded that the 1997-2002 AJCC recommendation that unilateral organ-confined tumors (pT2a) be separate category from bilateral (pT2b) should be eliminated as there was no significant recurrence-free survival between these pT2a and pT2b categories.

The present study evaluated unilateral pathologic stage pT2 tumors using a point-count method for tumor extent evaluation $(9,10)$ that is superior to the visual estimate used by Eichelberger and Cheng (3). Tumor volume can accurately be calculated using computer-assisted image analysis systems. Because this method is not feasible for routine clinical practice, other investigators have proposed alterna- 
tive simpler means of measuring tumor volume including diameter of largest tumor focus, number of tumor foci, number of involved blocks, percentage of blocks involved, use of a grid with $3.0 \mathrm{~mm}$ squares, or naked eye examination of the glass slides after the pathologist had circled all microscopically identifiable foci of carcinoma with a marking pen (the pathologist's percentage estimate) (13-18).

The method for evaluating tumor extent applied in this study is a simple one and accessible to all general pathologists $(9,10)$. It does not need any special device except a drawing on a sheet of paper. It is not time consuming because the pathologist draws on a sheet of paper the proportional area seen on the microscopic field at the same time he examines the slides. Considering that only a visual estimate of tumor extent provides important prognostic information after radical prostatectomy (14), the procedure used in this study seems to be superior because it includes a semi-quantitative point-count method represented by 8 equidistant points in each quadrant of the whole-mount transverse sections.

\section{CONCLUSIONS}

Using the 2002 TNM staging system, the majority of the totally embedded, serially sectioned, whole-mount surgical specimens of patients submitted to retropubic prostatectomy were pathologic stage pT2, however no stage pT2b tumors were identified (unilateral tumors that extended to more than half the area using a point-count method for tumor extent evaluation). Our results question the existence of a true pathologic stage pT2b.

\section{CONFLICT OF INTEREST}

None declared.

\section{REFERENCES}

1. International Union Against Cancer (UICC): TNM Classification of malignant tumours, 5th ed, Sobin LH,
Wittekind Ch (ed.), New York, Wiley-Liss. 1997; pp. 170-3.

2. International Union Against Cancer (UICC): TNM Classification of malignant tumours, 6th ed, Sobin LH, Wittekind Ch (ed.), New York, Wiley-Liss. 2002; pp. 184-7.

3. Eichelberger LE, Cheng L: Does pT2b cancer exist? Critical appraisal of the 2002 TNM classification of prostate carcinoma. Cancer. 2004; 100: 2573-6.

4. Mostofi, FK, Price EB Jr: Tumors of the Male Genital System, Atlas of Tumor Pathology, Second Series, Fascicle 8. Washington DC, Armed Forces Institute of Pathology. 1973; pp. 202-17.

5. Gleason DF, Mellinger GT: Prediction of prognosis for prostatic adenocarcinoma by combined histological grading and clinical staging. J Urol. 1974;111: 58-64.

6. Gleason DF: Histologic grading of prostate cancer: A perspective. Hum Pathol. 1992; 23: 273-9.

7. Epstein JI, Carmichael M, Walsh PC: Adenocarcinoma of the prostate invading the seminal vesicle: definition and relation of tumor volume, grade and margins of resection to prognosis. J Urol. 1993; 149: 1040-5.

8. Bostwick DG, Montironi R: Evaluating radical prostatectomy specimens: therapeutic and prognostic importance. Virchows Arch. 1997; 430: 1-16.

9. Billis A, Magna LA, Ferreira U: Correlation between tumor extent in radical prostatectomies and preoperative PSA, histological grade, surgical margins, and extraprostatic extension: Application of a new practical method for tumor extent evaluation. Int Braz J Urol. 2003; 29: 113-20.

10. Billis A, Freitas LL, Magna LA, Samara AB, Ferreira U: Prostate cancer with bladder neck involvement: Pathologic findings with application of a new practical method for tumor extent evaluation and recurrencefree survival after radical prostatectomy. Int Urol Nephrol. 2004; 36: 363-8.

11. Freedland SJ, Partin AW, Epstein JI, Walsh PC: Biochemical failure after radical prostatectomy in men with pathologic organ-confined disease: pT2a versus pT2b. Cancer. 2004; 100: 1646-9.

12. Rubin MA, Dash A, Wei JT, Dunn R, Sanda MG: Prostate cancer staging: Recommendations for modifying the 2002 AJCC pathology staging system based on accuracy in reflecting prognosis. Mod Pathol. 2004; 17 (suppl 1): 174A.

13. Cantrell BB, DeKlerk DP, Eggleston JC, Boitnott JK, Walsh PC: Pathological factors that influence prognosis in stage A prostatic cancer: the influence of extent versus grade. J Urol. 1981; 125: 516-20. 


\title{
Prostate Cancer Pathologic Stage pT2b
}

14. Humphrey PA, Vollmer RT: Percentage carcinoma as a measure of prostatic tumor size in radical prostatectomy tissues. Mod Pathol. 1997; 10: 326-33.

15. Renshaw AA, Chang H, D'Amico AV: Estimation of tumor volume in radical prostatectomy specimens in routine clinical practice. Am J Clin Pathol. 1997; 107: 704-8.

16. Renshaw AA, Richie JP, Loughlin KR, Jiroutek M, Chung A, D'Amico AV: Maximum diameter of prostatic carcinoma is a simple, inexpensive, and independent predictor of prostate-specific antigen failure in radical prostatectomy specimens. Validation in a co- hort of 434 patients. Am J Clin Pathol. 1999; 111: 6414.

17. Humphrey PA, Vollmer RT: Intraglandular tumor extent and prognosis in prostatic carcinoma: application of a grid method to prostatectomy specimens. Hum Pathol. 1990; 21: 799-804.

18. Carvalhal GF, Humphrey PA, Thorson P, Yan Y, Ramos CG, Catalona WJ: Visual estimate of the percentage of carcinoma is an independent predictor of prostate carcinoma recurrence after radical prostatectomy. Cancer. 2000; 89:1308-14.

Accepted after revision:

September 30, 2005

\author{
Correspondence address: \\ Dr. Athanase Billis \\ Dept de Anatomia Patológica \\ Fac. de Ciências Médicas - UNICAMP \\ Caixa Postal 6111 \\ Campinas, SP, 13084-971, Brazil \\ E-mail: athanase@fcm.unicamp.br
}

